\title{
Erratum to: Performance analysis and design of MIMO-OFDM system using concatenated forward error correction codes
}

\author{
Arun Agarwal ${ }^{1}$, Saurabh N. Mehta ${ }^{2}$
}

1. PhD Scholar, School of Electrical Engineering, Department of Information Technology, AMET University,

Tamil Nadu, Chennai 603112, India;

2. Department of Electronics and Telecommunication Engineering, Vidyalankar Institute of Technology,

Mumbai-400037, Maharashtra, India

(C) Central South University Press and Springer-Verlag GmbH Germany 2017

Erratum to: J. Cent. South Univ. (2017) 24: 1322-1343

DOI: $10.1007 / \mathrm{s} 11771-017-3537-2$

Figures 14-25 in the original version of the article are incorrect and they should be replaced as follows:
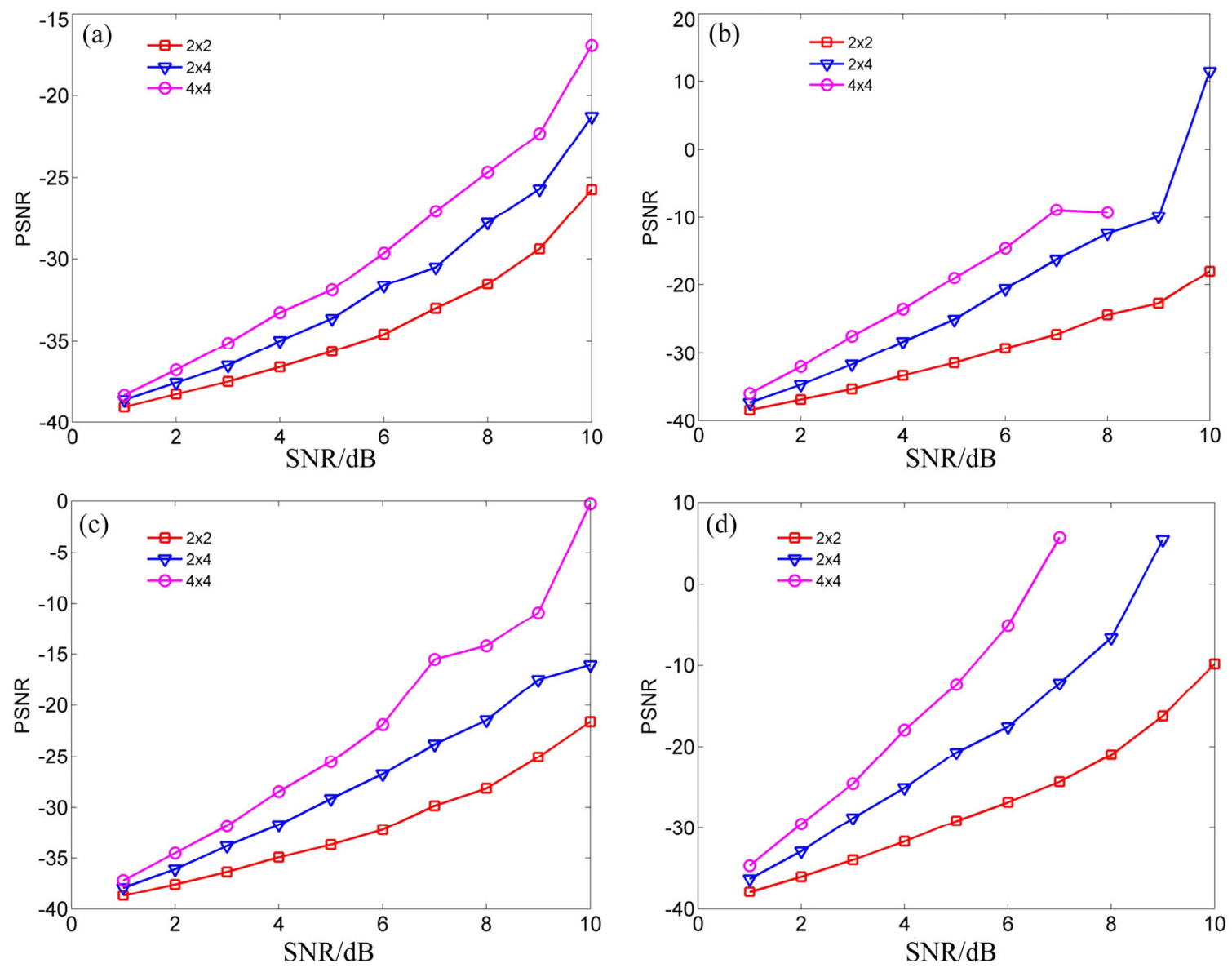

Fig. 14 PSNR analysis of MIMO-OFDM system with BPSK modulation and FEC schemes in AWGN environment: (a) Only convolutional coding, CC; (b) LDPC concatenated with convolutional coding, LDPC+CC; (c) RSC concatenated with convolutional coding, RSC+CC; (d) Turbo codes concatenated with convolutional coding 

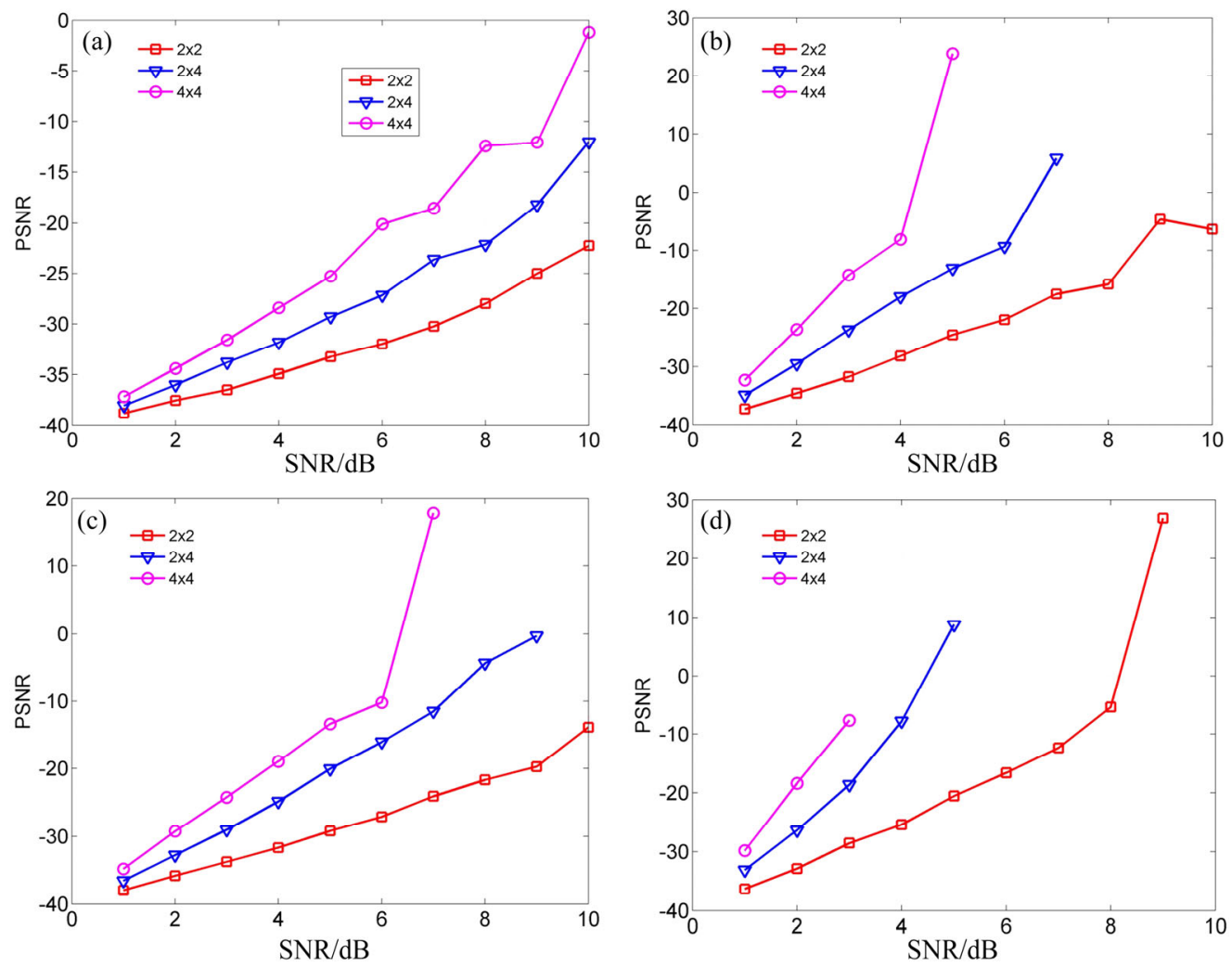

Fig. 15 PSNR analysis of MIMO-OFDM system with QPSK modulation and FEC schemes in AWGN environment: (a) Only convolutional coding, CC; (b) LDPC concatenated with convolutional coding, LDPC $+\mathrm{CC}$; (c) RSC concatenated with convolutional coding, RSC $+\mathrm{CC}$; (d) Turbo codes concatenated with convolutional coding
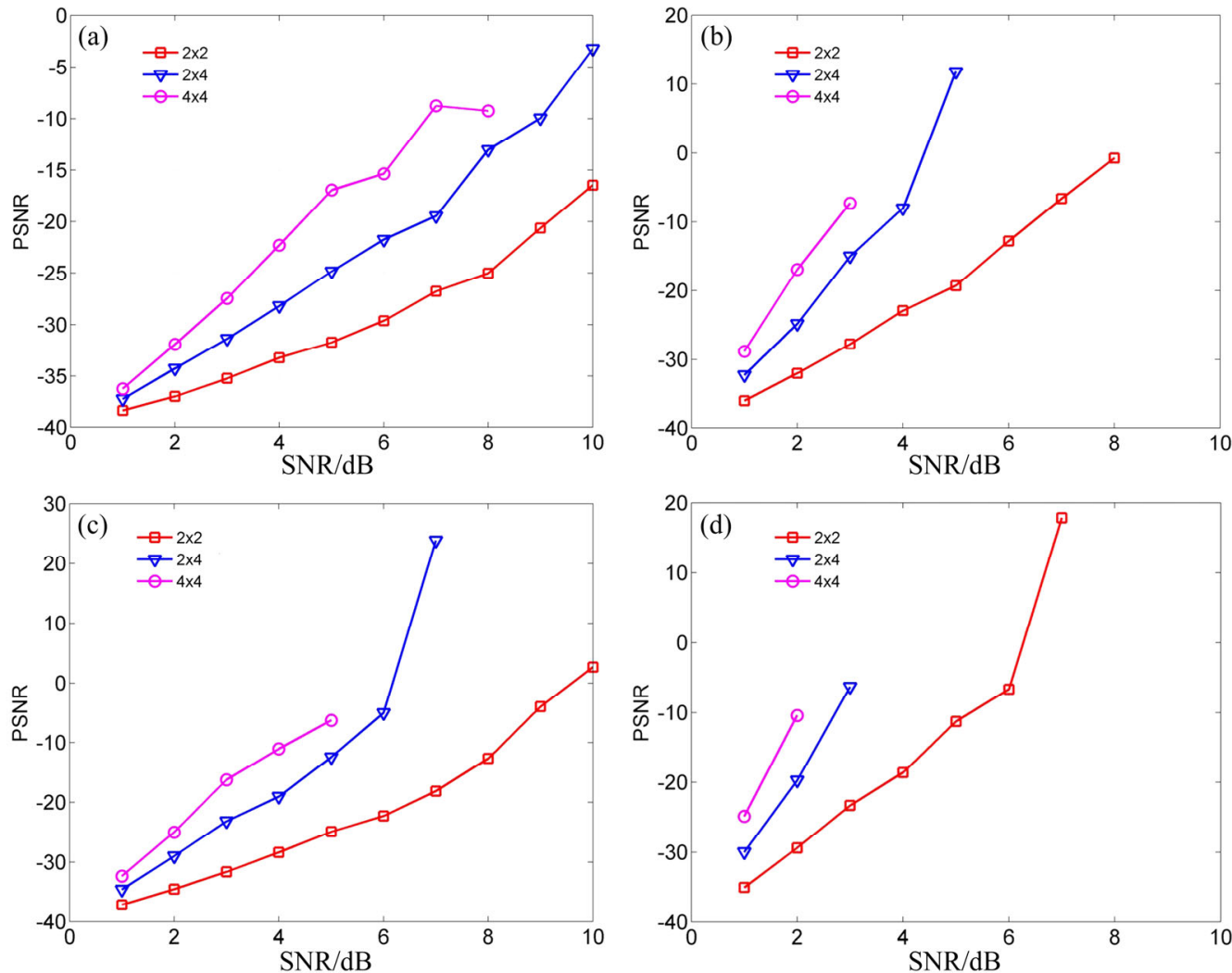

Fig. 16 PSNR analysis of MIMO-OFDM system with QAM-16 modulation and FEC schemes in AWGN environment: (a) Only convolutional coding, CC; (b) LDPC concatenated with convolutional coding, LDPC $+\mathrm{CC}$; (c) RSC concatenated with convolutional coding, RSC $+\mathrm{CC}$; (d) Turbo codes concatenated with convolutional coding 

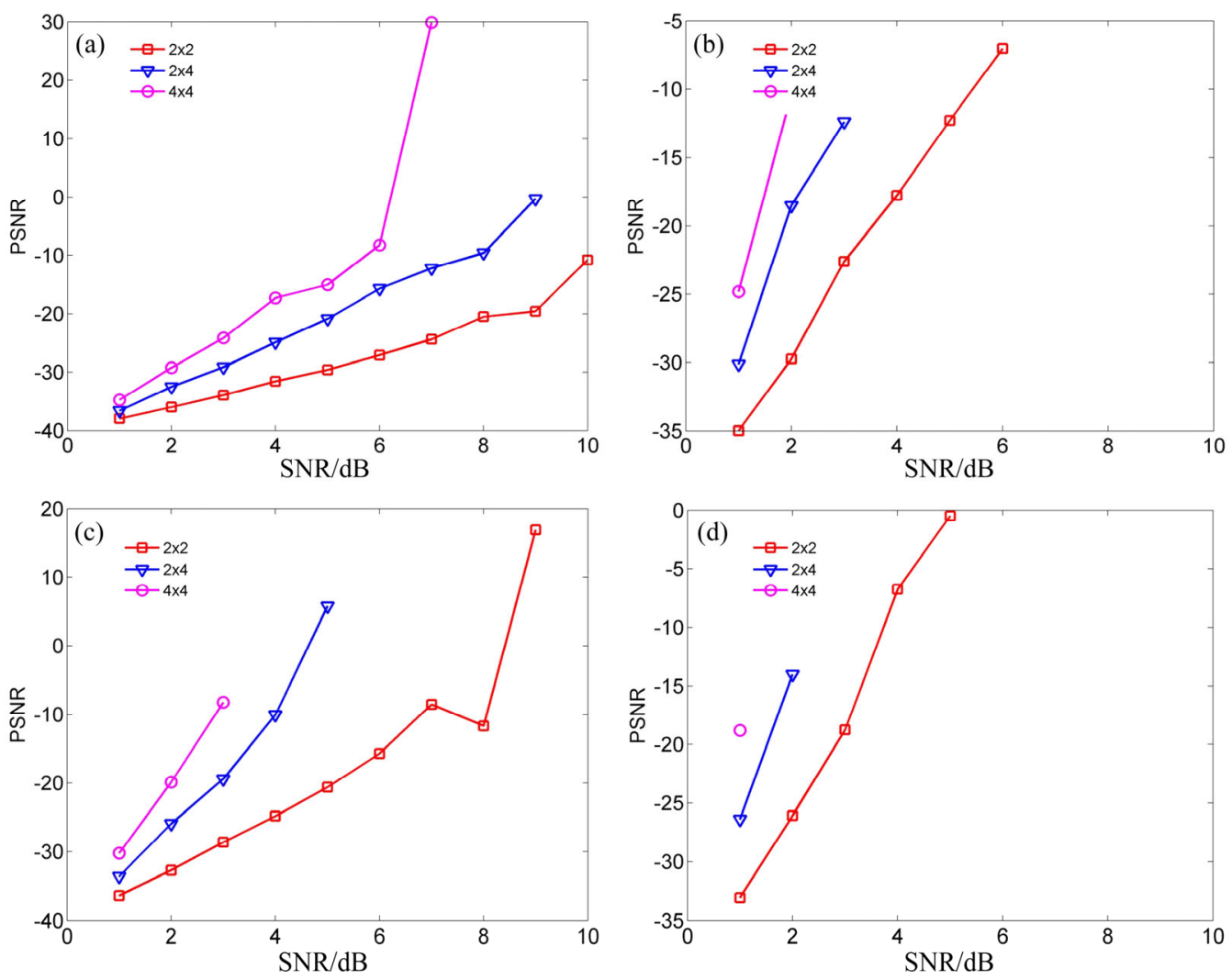

Fig. 17 PSNR analysis of MIMO-OFDM system with QAM-64 modulation and FEC schemes in AWGN environment: (a) Only convolutional coding, CC; (b) LDPC concatenated with convolutional coding, LDPC+CC; (c) RSC concatenated with convolutional coding, RSC $+\mathrm{CC}$; (d) Turbo codes concatenated with convolutional coding
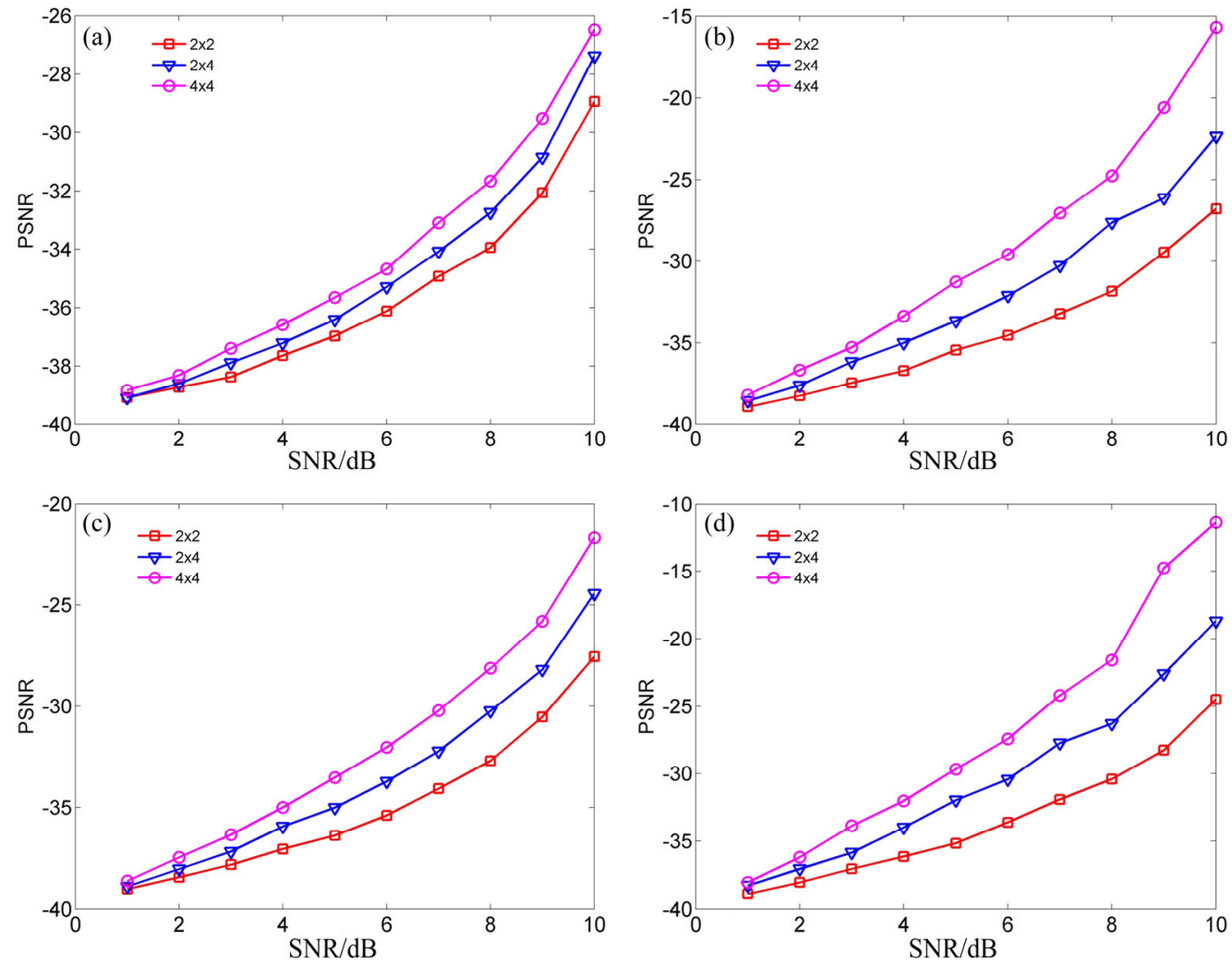

Fig. 18 PSNR analysis of MIMO-OFDM system with BPSK modulation and FEC schemes in Rayleigh environment: (a) Only convolutional coding, CC; (b) LDPC concatenated with convolutional coding, LDPC+CC; (c) RSC concatenated with convolutional coding, RSC $+\mathrm{CC}$; (d) Turbo codes concatenated with convolutional coding 

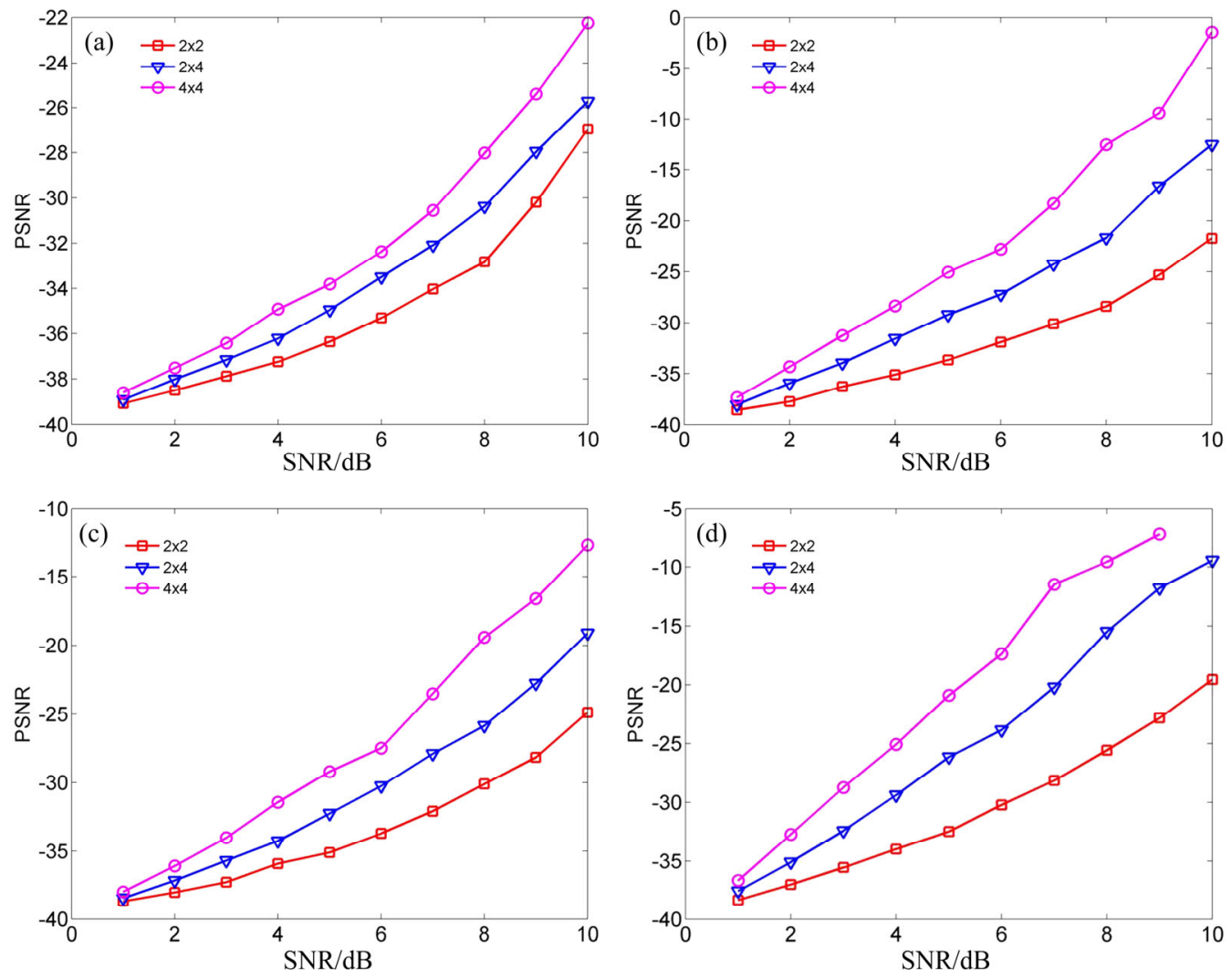

Fig. 19 PSNR analysis of MIMO-OFDM system with QPSK modulation and FEC schemes in Rayleigh environment: (a) Only convolutional coding, CC; (b) LDPC concatenated with convolutional coding, LDPC+CC; (c) RSC concatenated with convolutional coding, RSC $+\mathrm{CC}$; (d) Turbo codes concatenated with convolutional coding
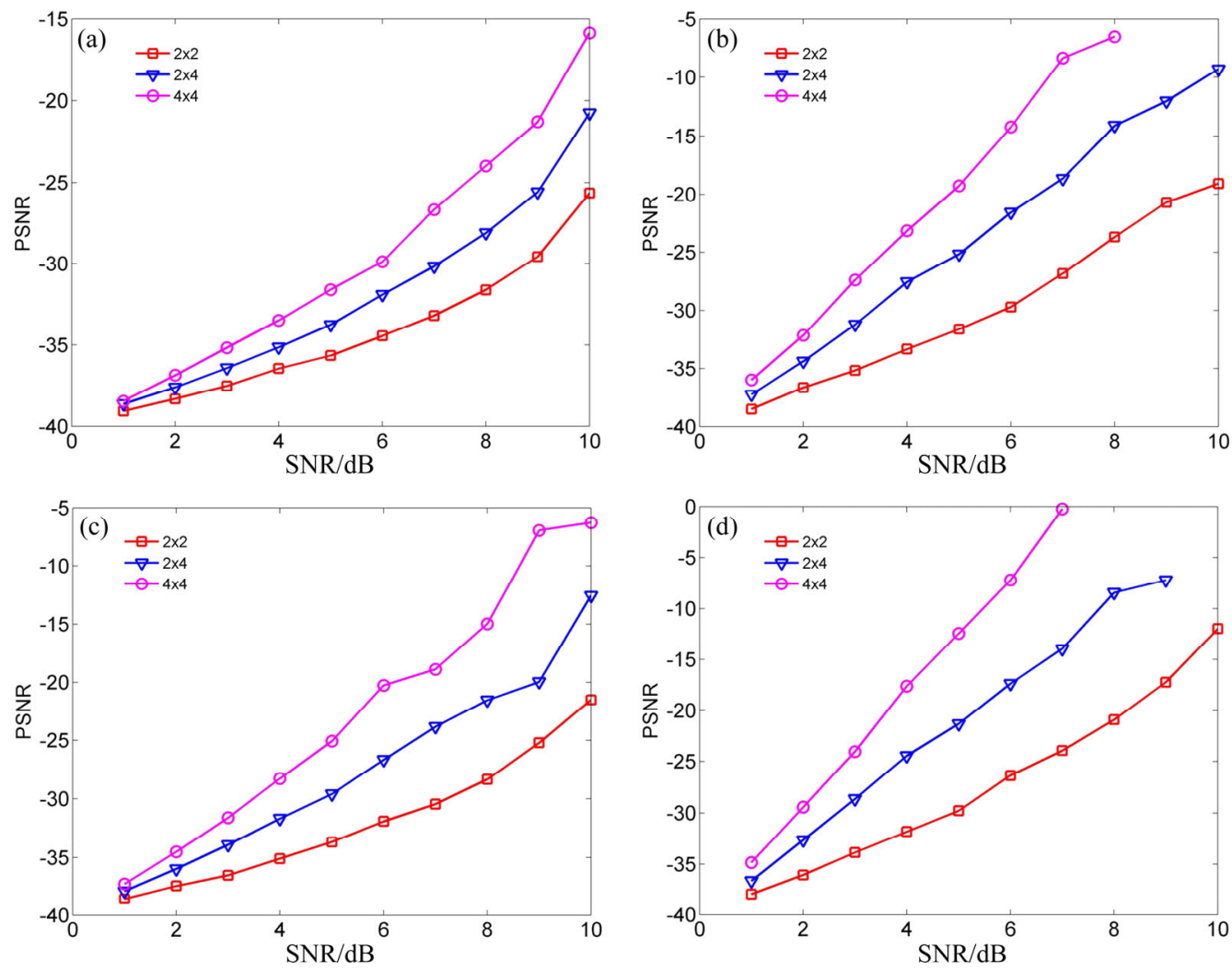

Fig. 20 PSNR analysis of MIMO-OFDM system with QAM-16 modulation and FEC schemes in Rayleigh environment: (a) Only convolutional coding, CC; (b) LDPC concatenated with convolutional coding, LDPC $+\mathrm{CC}$; (c) RSC concatenated with convolutional coding, RSC $+\mathrm{CC}$; (d) Turbo codes concatenated with convolutional coding 

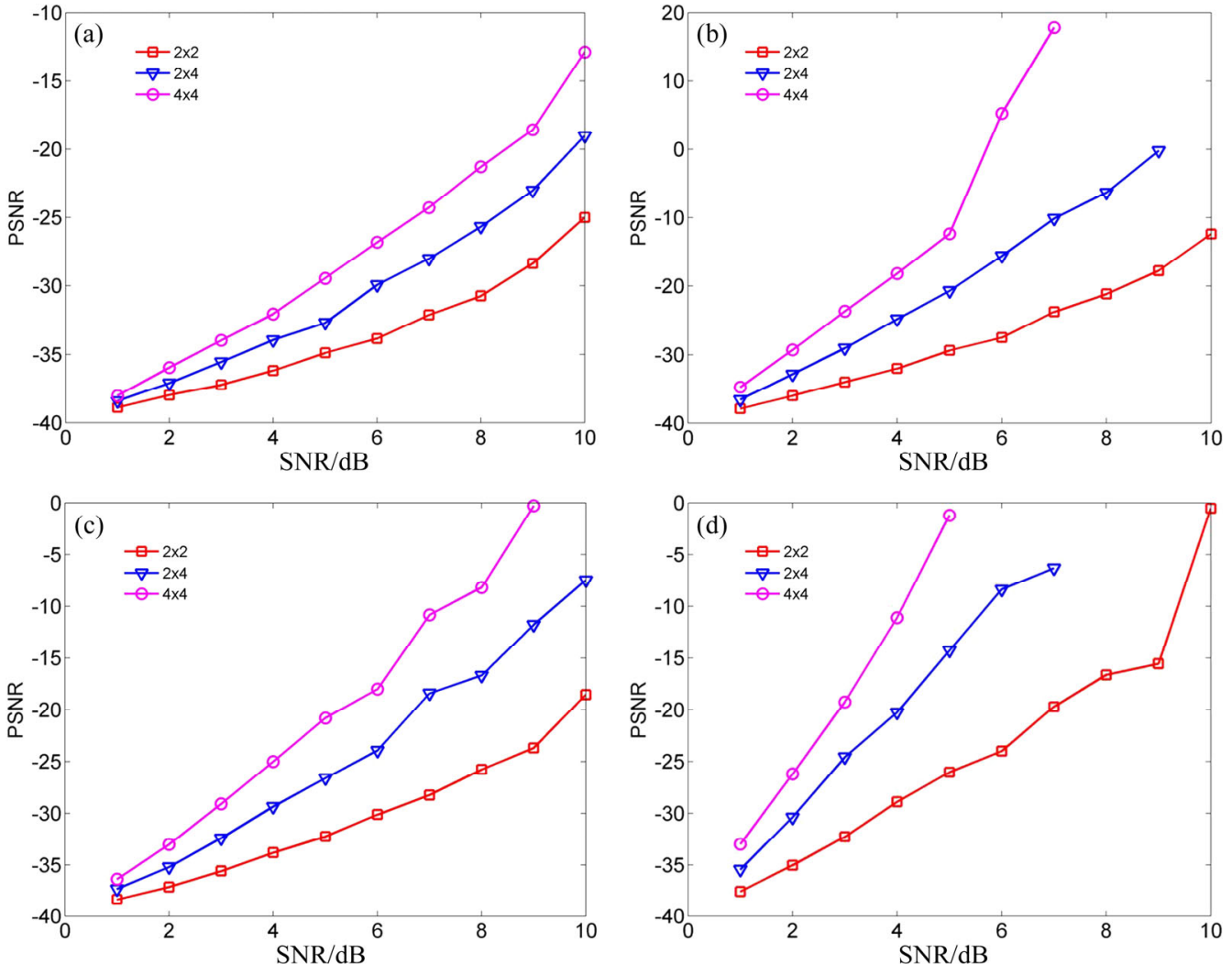

Fig. 21 PSNR analysis of MIMO-OFDM system with QAM-64 modulation and FEC schemes in Rayleigh environment: (a) Only convolutional coding, CC; (b) LDPC concatenated with convolutional coding, LDPC $+\mathrm{CC}$; (c) RSC concatenated with convolutional coding, RSC+CC; (d) Turbo codes concatenated with convolutional coding
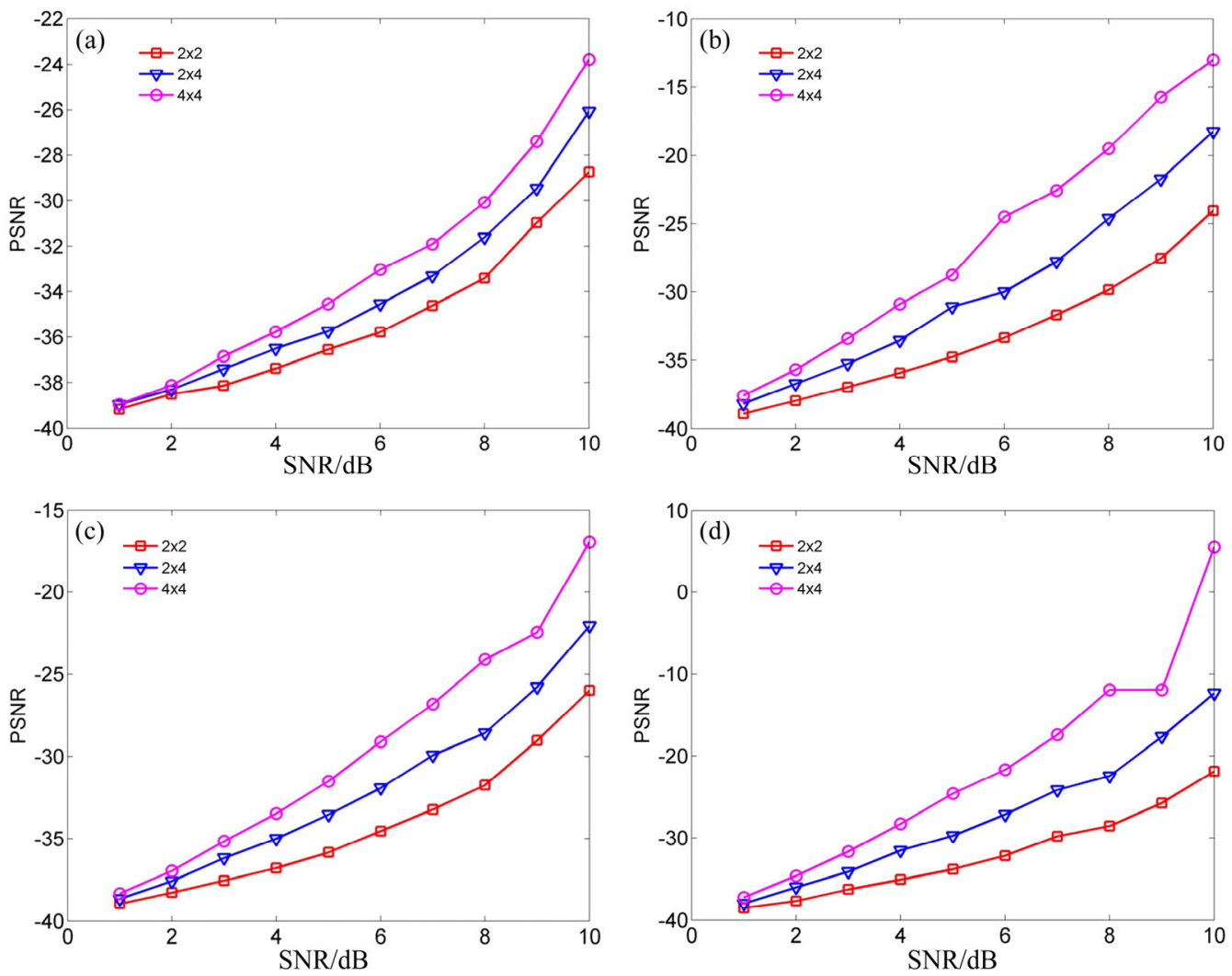

Fig. 22 PSNR analysis of MIMO-OFDM system with BPSK modulation and FEC schemes in Rician environment: (a) Only convolutional coding, CC; (b) LDPC concatenated with convolutional coding, LDPC+CC; (c) RSC concatenated with convolutional coding, RSC $+\mathrm{CC}$; (d) Turbo codes concatenated with convolutional coding 

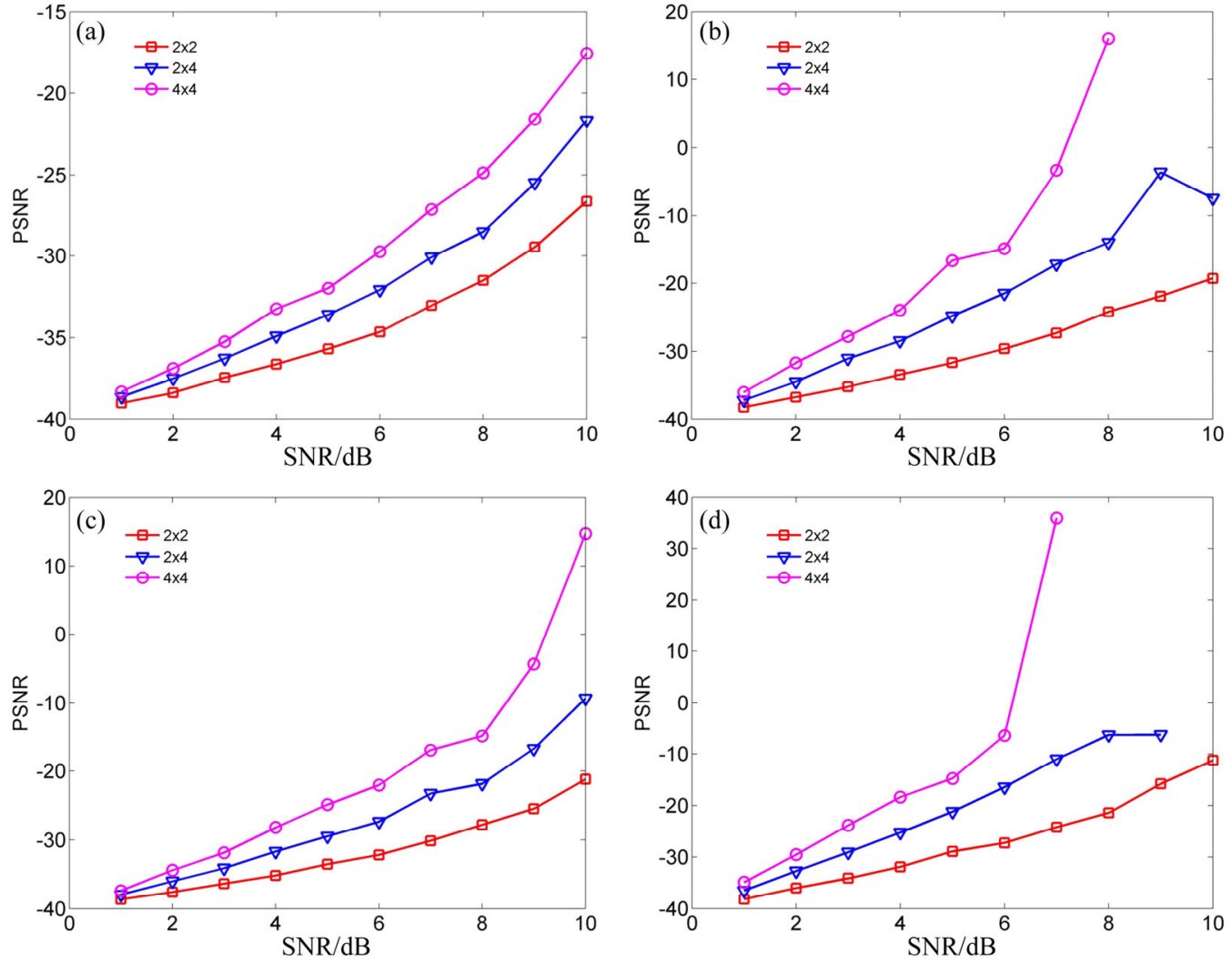

Fig. 23 PSNR analysis of MIMO-OFDM system with QPSK modulation and FEC schemes in Rician environment: (a) Only convolutional coding, CC; (b) LDPC concatenated with convolutional coding, LDPC+CC; (c) RSC concatenated with convolutional coding, RSC+CC; (d) Turbo codes concatenated with convolutional coding
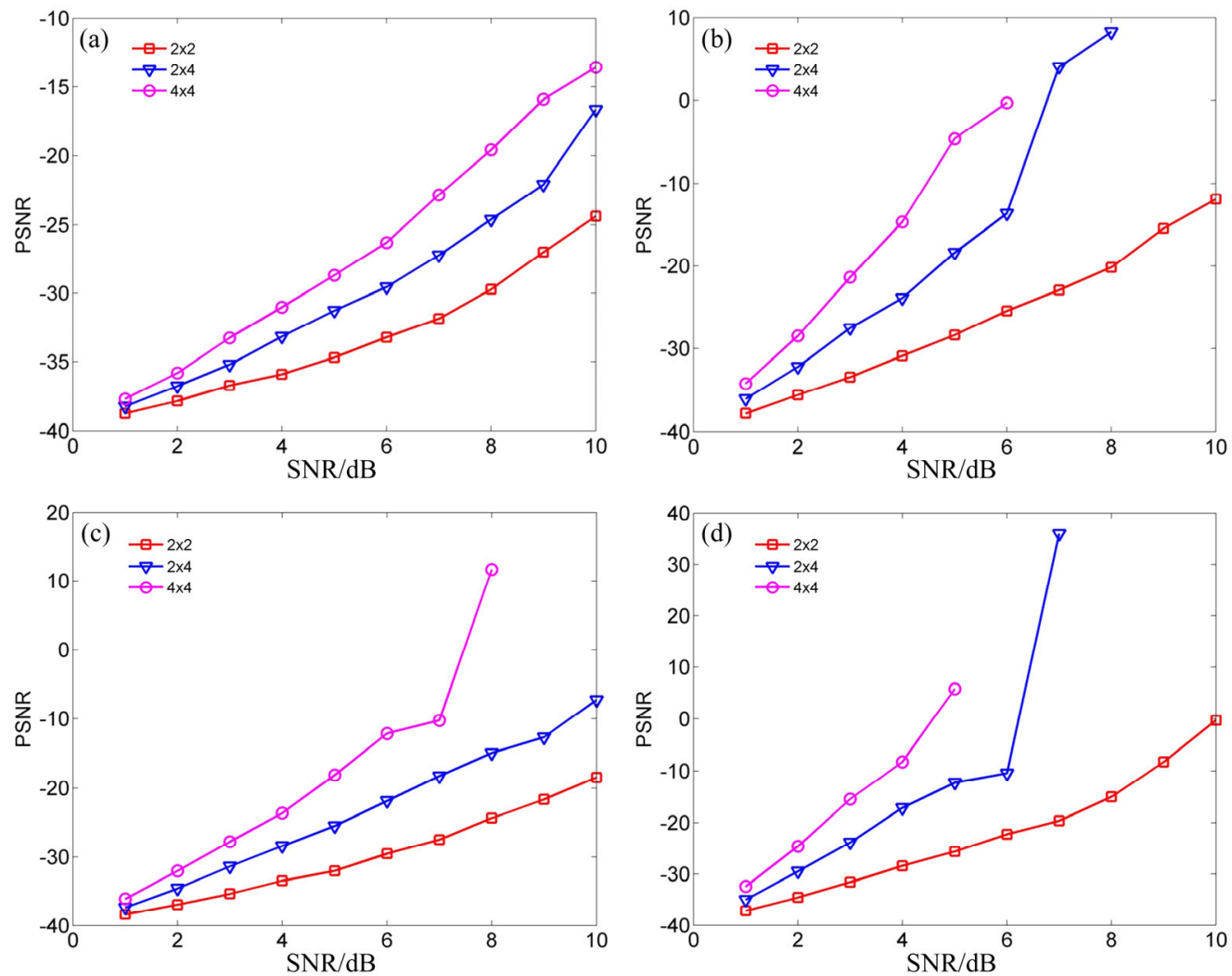

Fig. 24 PSNR analysis of MIMO-OFDM system with QAM-16 modulation and FEC schemes in Rician environment: (a) Only convolutional coding, CC; (b) LDPC concatenated with convolutional coding, LDPC $+\mathrm{CC}$; (c) RSC concatenated with convolutional coding, RSC $+\mathrm{CC}$; (d) Turbo codes concatenated with convolutional coding 

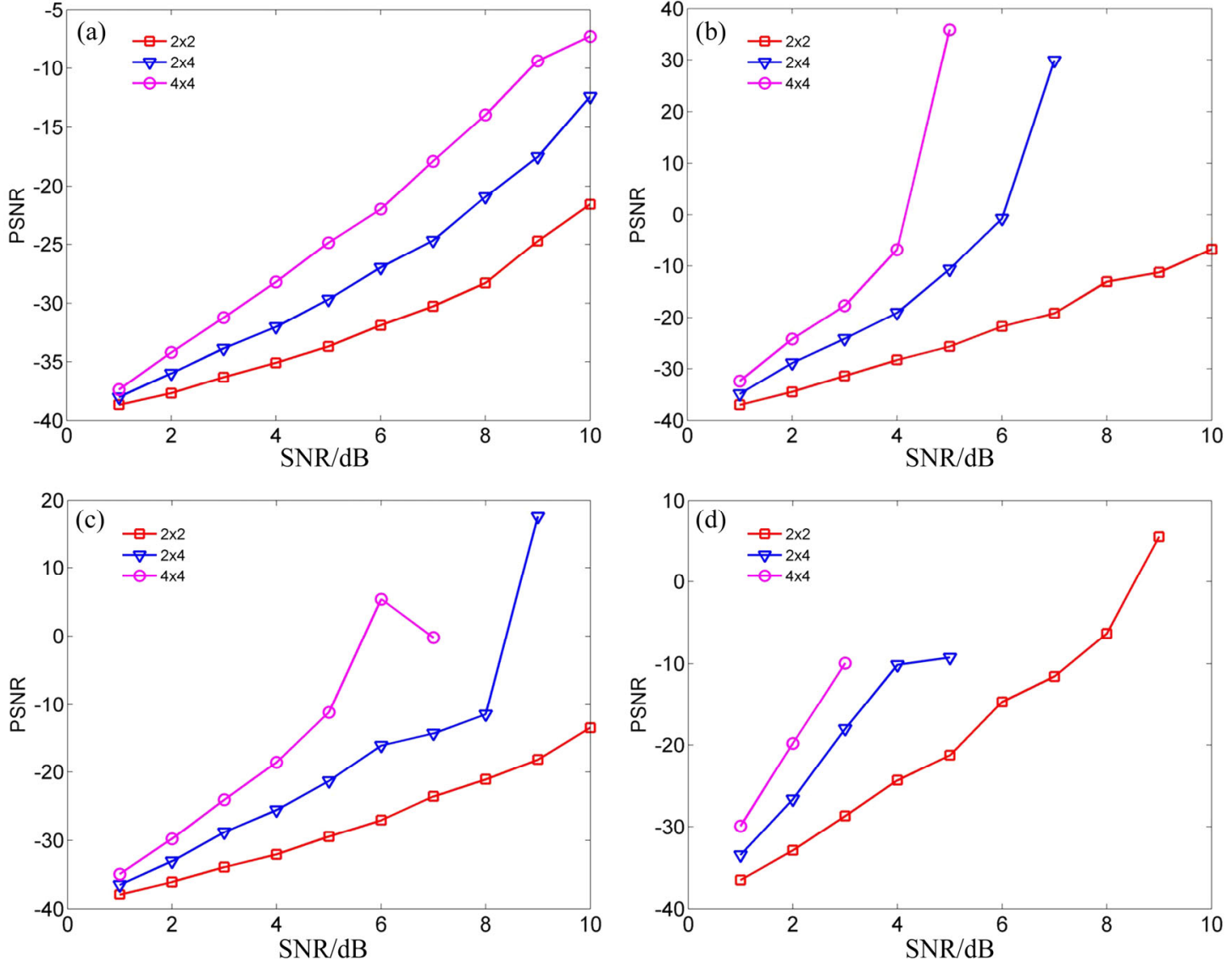

Fig. 25 PSNR analysis of MIMO-OFDM system with QAM-64 modulation and FEC schemes in Rician environment: (a) Only convolutional coding, CC; (b) LDPC concatenated with convolutional coding, LDPC $+\mathrm{CC}$; (c) RSC concatenated with convolutional coding, RSC+CC; (d) Turbo codes concatenated with convolutional coding 